\title{
Superconducting single-photon detector for integrated waveguide spectrometer
}

\author{
Alexander Korneev ${ }^{1,2, *}$, Vadim Kovalyuk ${ }^{1}$, Pavel $\mathrm{An}^{1}$, Alexander Golikov ${ }^{1}$, Evgeniya \\ Zubkova $^{2,1}$, Simone Ferrari ${ }^{3}$, Oliver Kahl ${ }^{3}$, Wolfram Pernice ${ }^{3}$, and Gregory Goltsman ${ }^{1,2}$ \\ ${ }^{1}$ Moscow State Pedagogical University, 119991 Moscow, Russia \\ ${ }^{2}$ National Research University Higher School of Economics, 101000 Moscow, Russia \\ ${ }^{3}$ University of Münster, Institute of Physics, 48149 Münster, Germany
}

\begin{abstract}
We present our recent achievements in the development of an on-chip spectrometer consisting of arrayed waveguide grating made of $\mathrm{Si}_{3} \mathrm{~N}_{4}$ waveguides and $\mathrm{NbN}$ superconducting single-photon detector.
\end{abstract}

Superconducting single-photon detectors (SSPD) [1,2] became recently a device of choice in many applications ranging from quantum dots characterization to quantum cryptography and long distance space communication. The key factor of their success is superior performance: above $90 \%$ detection efficiency, very low dark count rate, picosecond timing jitter and high photon count rate. The superb performance makes SSPD especially attractive for advanced applications in quantum optics.

At the same time, there is a long-term trend in practical quantum optics to switch to optical integrated circuits on a single chip in which the light is delivered from the source to the detector by the dielectric waveguides. The waveguides were realized by means of several materials platforms, such as silicon on insulator ( $\mathrm{SOI}$ ), gallium arsenide (GaAs), silicon nitride $\left(\mathrm{Si}_{3} \mathrm{~N}_{4}\right)$ and polycrystalline diamond.

In [3] integration of SSPD with dielectric optical waveguides was demonstrated featuring near unity absorption for the wave travelling in the waveguide. At the same time, due to shorter nanostrip length compared to the traditional meander-shaped SSPD it features also shorter dead time and thus is capable of higher photon counting rates. Advanced photon detection capabilities offered by SSPD make such integrated circuits feasible for applications in optical signal processing, optical sensing and linear optical quantum computing.

In this paper, we present an approach to the realization of an on-chip spectrometer based arrayed waveguide grating (AWG) demultiplexer integrated with SSPD as shown in Fig. 1(a). The AWG is patterned from silicon nitride $\mathrm{Si}_{3} \mathrm{~N}_{4}$ on silicon substrate with buried oxide by e-beam lithography. The input light is split by the AWG in several output channels, each with its own wavelength. We fabricated two types of eight-channel devices operating near the wavelength of $1550 \mathrm{~nm}$ and $740 \mathrm{~nm}$. The main characteristics of the devices, including the free spectral range and channel width are presented in Table 1.

Each output waveguide is integrated with the U-shaped SSPD. In order to realize

\footnotetext{
* Corresponding author: akorneev@ rplab.ru
} 
optimal detector performance, the detector layout which produced the best results at $1550 \mathrm{~nm}$ during the previous characterization was chosen. All integrated SNSPDs on the AWGs were therefore $80 \mathrm{~nm}$ wide, $80 \mu \mathrm{m}$ long with a gap of $100 \mathrm{~nm}$ between the stripes. The whole device is operated at $1.7 \mathrm{~K}$ temperature providing best performance of the SSPD. Fig. 1(b) shows on-chip detection efficiency (probability to detect photons of a wave in the waveguide, the losses at the input couplers are not taken into account) and dark count rate of the SSPD as a function of its bias current normalized to the critical current at which superconducting state is destroyed.
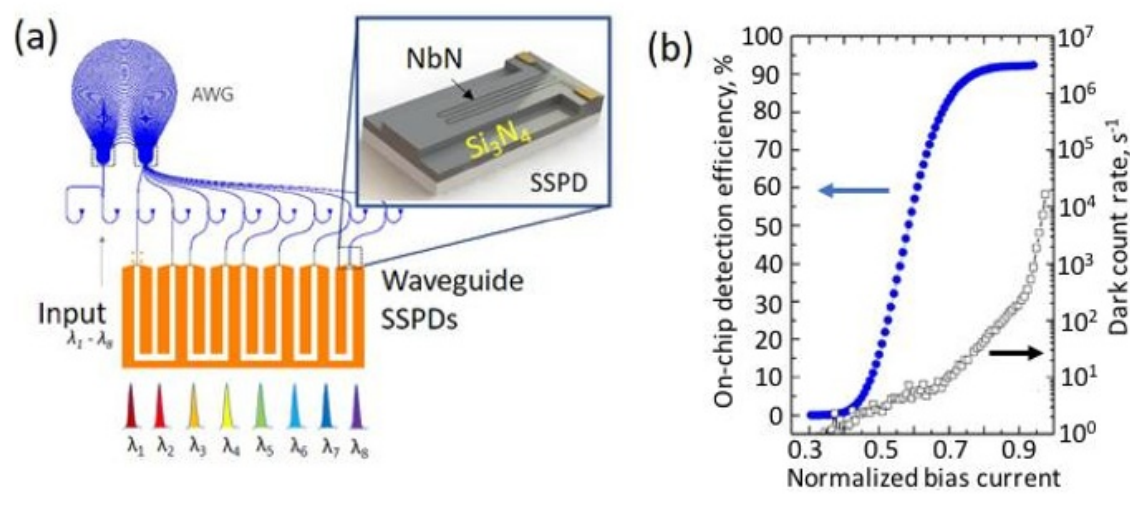

Fig. 1. (a) Schematics of AWG demultiplexer integrated with SSPD; (b) on-chip detection efficiency and dark counts rate of SSPD as functions of SSPD normalized bias current, a ratio of bias current $I_{b}$ to the critical current $I_{c}$. Maximum on-chip detection efficiency can be as high as $92 \%$ at below 100 dark counts per second.

Table 1. AWG Parameters for the spectrometer.

\begin{tabular}{|c|c|c|c|c|}
\hline $\begin{array}{c}\text { Central } \\
\text { wavelength, nm }\end{array}$ & $\begin{array}{c}\text { Diffraction } \\
\text { order }\end{array}$ & $\begin{array}{c}\text { Free spectral } \\
\text { range (FSR), nm }\end{array}$ & $\begin{array}{c}\text { Transmission } \\
\text { window, nm }\end{array}$ & $\begin{array}{c}\text { Channel FWHM, } \\
\mathrm{nm}\end{array}$ \\
\hline 1550 & 54 & 24 & 16 & 2,2 \\
\hline 740 & 11 & 60 & 45 & 6,4 \\
\hline
\end{tabular}

The losses in the waveguide circuit reduced the on-chip detection efficiency to $19 \% \pm 6 \%$ and $21 \pm 14 \%$ for wavelengths of $1550 \mathrm{~nm}$ and $740 \mathrm{~nm}$, respectively, as well as coupling losses restrict the system detection efficiency to the 2\%@1550 nm and 0.42\%@740 nm. By optimizing the waveguide circuit and using more efficient coupling with the optical fiber, the system detection efficiency can be significantly improved in the future.

This work was supported by Russian Science Foundation project 16-19-10633.

\section{References}

1. G. Gol'tsman, O. Okunev, G. Chulkova et al., Appl. Phys. Lett. 79, 705 (2001)

2. C. M Natarajan, M. G Tanner, R. H. Hadfield, Supercond. Sci. Technol. 25, 063001 (2012)

3. W. Pernice, C. Schuck, O. Minaeva et al., Nat. Commun. 3, 1325 (2012) 